\title{
Calibrationless Parallel MRI with Joint Total Variation Regularization
}

\author{
Chen Chen, Yeqing Li, and Junzhou Huang \\ University of Texas at Arlington, TX, USA 76019
}

\begin{abstract}
In this paper, a calibrationless method is proposed for parallel magnetic resonance imaging (pMRI). It is motivated by the observation that the gradients of the aliased images are jointly sparse. Therefore, the pMRI problem can be formulated as a joint total variation regularization task. The field of view is finally obtained via a sum of square approach. We develop an iterative algorithm to efficiently solve this problem. Experiments on pMRI datasets demonstrate that our method outperforms the state-of-the-art pMRI methods even when they can achieve sufficient calibration data, and far better than existing calibrationless pMRI algorithms. Clinic MR applications could benefit from this method even when accurate calibration is limited or not possible at all.
\end{abstract}

\section{Introduction}

It is routine to accelerate MRI with parallel technique in clinic applications by using multiple receiver coils to acquire undersampled k-space data. The coils are installed around the patient at different locations. Thus, the MR signals are scanned with different spatial sensitivities. If estimated precisely, the sensitivity information could be used to interpolate the missing data due to undersampling.

The data acquired by each receiver coil corresponds to an aliased image. Based on the way to reconstruct each aliased image, existing methods can be classified broadly in two types: a) image domain methods such as SENSE [1] and JSENSE [2, which directly transform the undersampled data to aliased images and then unfold them to the field of view (FOV) via SENSE encoding; b) frequency domain methods such as GRAPPA [3] and SPIRiT [4, which interpolate the missing data to fill the full k-space and then transform them to aliased images. For the image domain methods, the sensitivity map is required to be estimated. Any noise or inaccuracy in the sensitivity map will be amplified significantly and result in visible artifacts in the FOV. For the frequency domain methods, the interpolation weights need to be calibrated with sufficient auto-calibration signals (ACS). However, the efficiency or feasibleness of calibration is limited in many applications such as dynamic MRI and non-Cartesian imaging. Without sufficient ACS, the performance of all these methods and their improved versions [5] 6] can not be guaranteed.

To avoid calibration, an algorithm CaLMMRI is proposed recently [7]. It is based on compressive sensing MRI (CSMRI) 8] scheme to reconstruct MR image from undersampled data without the need for the sensitivity information. Because all the aliased images represent the same anatomical cross section, 
they are jointly reconstructed by exploiting the joint sparsity among them. The FOV is obtained by the sum of square (SoS) approach with the reconstructed aliased images. However, their assumption on the appearance similarity among aliased images does not hold strictly due to the sensing difference caused by the coil locations. This makes their algorithm still hard to be comparable to the state-of-the-art calibration methods such as GRAPPA and SPIRiT. As we are interested in calibrationless pMRI, CSSENSE [5] could also be viewed as a calibrationless method if the SENSE encoding step is replaced by SoS. Compared to CaLMMRI, CSSENSE can only reconstruct each aliased image coil-by-coil. No spatial prior knowledge in the data other than sparsity is utilized to improve the reconstruction. Moreover, all these CS based MRI methods [7] [5] strongly rely on the incoherence of the sampling matrix [9] [10, which can not be always guaranteed in MR applications.

In this paper, we propose a new pMRI method called Joint Total Variation MRI (JTVMRI), which is an extension of the total variation (TV) [1] regularization for ill-posed problem. Unlike CaLMMRI, we do not assume the images of the different coils have similar appearance but only assume their gradients are similar. Since MR images are often piecewise smooth, most of the gradients are approximate to zeros. The non-zero gradients only appear on the edges, which is consistent in all the aliased images of different coils. An efficient algorithm is developed to efficiently solve the ill-posed pMRI problem. Our method does not require any calibration step and can be applied on arbitrary sampling which is not available for GRAPPA and SPIRiT. Extensive experiments demonstrate that the proposed method is far better than CaLMMRI. And more importantly, it outperforms the state-of-the-art auto-calibration method such as GRAPPA and SPIRiT, which makes calibrationless pMRI much feasible than before.

\section{Related Work}

\subsection{CSSENSE}

CSSENSE [5] is a hybrid approach, which apply CSMRI 4] to reconstruct the aliased images and the second step is to unfold them to full FOV with SENSE encoding [1]. Because we are interested in calibrationless pMRI in this paper, we consider it as a calibrationless CSMRI method, where the second step replaced with SoS. The problem to reconstruct one aliased image $x_{c}$ is:

$$
x_{c}=\arg \min _{x_{c}}\left\{\frac{1}{2}\left\|F x_{c}-b_{c}\right\|_{2}^{2}+\alpha\left\|x_{c}\right\|_{T V}+\beta\left\|\Phi x_{c}\right\|_{1}\right\} \quad c=1,2, \ldots, C
$$

where $C$ denotes the total number of coils; $\Phi$ denotes the wavelet transform; $F$ is the undersampled Fourier operator and $b_{c}$ is the undersampled k-space data for coil $c ; \alpha, \beta$ are two parameters. $\|x\|_{T V}=\sum_{i} \sqrt{\left(\nabla_{1} x_{i}\right)^{2}+\left(\nabla_{2} x_{i}\right)^{2}}$ for an image of $N$ pixels, $i=1, \ldots, N$, where $\nabla_{1}$ and $\nabla_{2}$ denote the forward finite difference operators on the first and second coordinates. Conjugate gradient method (CG) [4] is applied to solve this problem. No structured prior information is utilized and each image can only be reconstructed separately. 


\subsection{CaLMMRI}

CaLMMRI 7] is a CSMRI 4] approach for pMRI. It reconstructs the aliased images of all coils simultaneously with joint sparsity and the FOV is obtained via the SoS scheme.

$$
X=\arg \min _{X}\|\Phi X\|_{2,1} \quad \text { s.t. } \quad \sum_{c=1}^{C}\left\|F X(:, c)-b_{c}\right\|_{2}^{2}<\varepsilon
$$

where $X=\left[x_{1}, x_{2}, \ldots, x_{C}\right]$ is the images of all coils. The $\ell_{2,1}$ norm is the summation of each row's $\ell_{2}$ norm. Problem (2) is solved by SPGL1[12 in their method. It is a joint reconstruction method as ours. However, as discussed above, their assumption of the similar appearance among images of all coils can not be strictly satisfied in practice. This results in its relatively lower performance and makes it unable to be comparable to the state-of-the-art auto-calibration methods when sufficient ACS is available.

\section{Algorithm}

Different from CaLMMRI, we only assume the gradients of aliased images from all the coils are jointly sparse. Figure 1 demonstrates the appearances and gradients of two images from different coils. We could observe that the appearances are only similar at the center area. However, as MR images are often piecewise smooth, the gradients are not only very sparse but also jointly sparse. The non-zero gradients tend to be at the same positions across different images, that is, on the edges. This assumption holds at almost entire image areas. As an extension of TV regularization, the pMRI reconstruction is therefore formulated as a JTV reconstruction:

$$
X=\arg \min _{X}\left\{\frac{1}{2} \sum_{c=1}^{C}\left\|F X(:, c)-b_{c}\right\|_{2}^{2}+\alpha\|X\|_{J T V}\right\}
$$

where $\|X\|_{J T V}=\sum_{i=1}^{N} \sqrt{\sum_{c=1}^{C}\left(\left(\nabla_{1} X_{i c}\right)^{2}+\left(\nabla_{2} X_{i c}\right)^{2}\right)}$. The JTV is also known as vectorial TV [13] for color image processing. Problem (3) can be solved by proximal gradient method and accelerate by FISTA [14]. The whole algorithm is summarized in Algorithm 1, where $f(X)=\frac{1}{2} \sum_{c=1}^{C}\left\|F X(:, c)-b_{c}\right\|_{2}^{2}, \nabla f(X)$ denotes its gradient and $L$ is its Lipschitz constant.

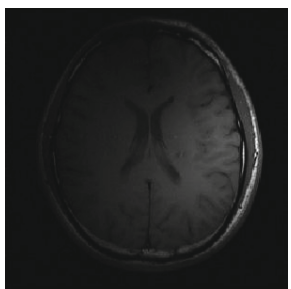

(a)

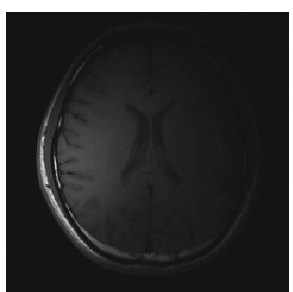

(b)

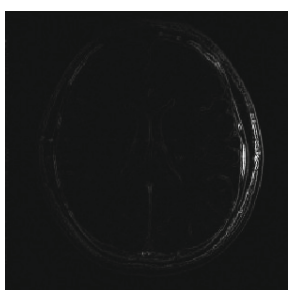

(c)

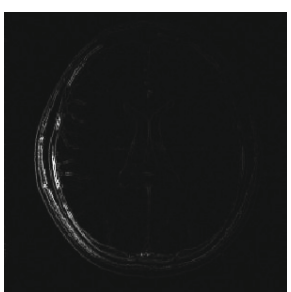

(d)

Fig. 1. The appearances and gradients of two aliased images from different coils. (a) and (b): appearances. (c) and (d): magnitude of gradients. 
The key problem is thus how to efficiently solve the JTV denoising problem in the second step:

$$
X=\arg \min _{X}\left\{\frac{1}{2}\|X-B\|^{2}+\lambda\|X\|_{J T V}\right\}
$$

where $\lambda=\alpha / L$. For convenience of solving (4), $X$ and $B$ are now reshaped to $m \times n \times C$ where $m, n$ denote the number of rows and the number of columns of an image. Following previous works [15, we consider a dual method for (4). Let $P \in \mathbb{R}^{(m-1) \times n \times C}, Q \in \mathbb{R}^{m \times(n-1) \times C}$ and they satisfied:

$$
\sum_{c=1}^{C}\left(P_{i, j, c}^{2}+Q_{i, j, c}^{2}\right) \leq 1, \quad \forall i, j, \quad\left|P_{i, n, c}\right| \leq 1,\left|Q_{m, j, c}\right| \leq 1 \quad \forall i, j, c
$$

A linear operator is defined as $\mathcal{L}(P, Q)_{i, j, c}=P_{i, j, c}-P_{i-1, j, c}+Q_{i, j, c}-Q_{i, j-1, c}$ and the corresponding inverse operator is defined as $\mathcal{L}^{T}(X)=(P, Q)$ with $P_{i, j, c}=X_{i, j, c}-X_{i+1, j, c}, Q_{i, j, c}=X_{i, j, c}-X_{i, j+1, c}$.
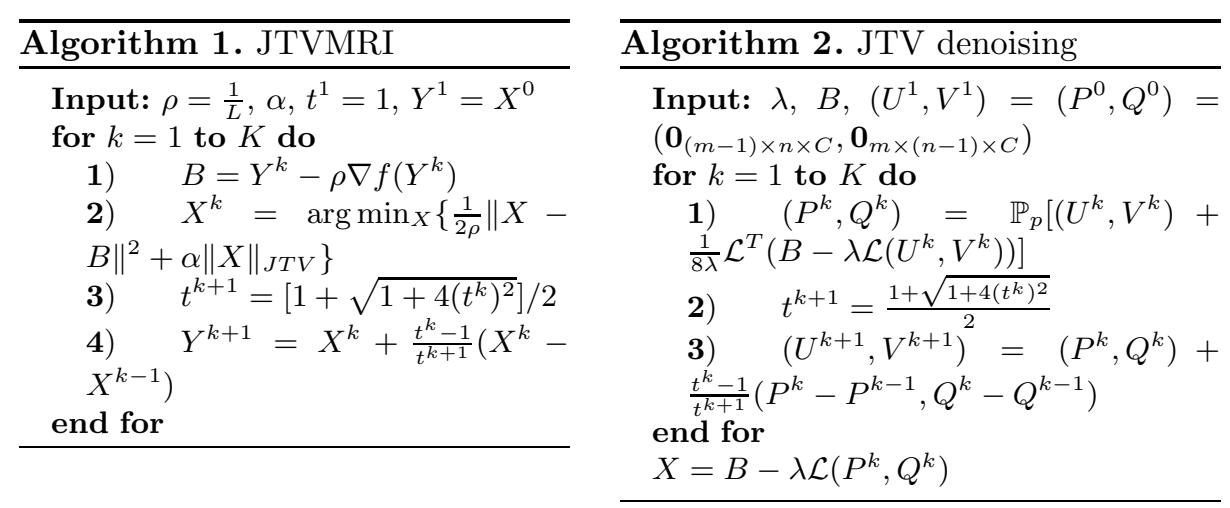

Therefore, the optimal solution for problem (4) is $X=B-\lambda \mathcal{L}\left(P^{*}, Q^{*}\right)$ [15], where $P^{*}, Q^{*}$ is the optimal solution for

$$
\min _{P, Q}\left\{h(P, Q)=\frac{1}{2}\|B-\lambda \mathcal{L}(P, Q)\|_{F}^{2}\right\}
$$

Note that the gradient of $h(P, Q)=-\lambda(B-\lambda \mathcal{L}(P, Q))$. Therefore problem (6) can be solved by FISTA and summarized in Algorithm 2 .

The projection operator $\mathbb{P}_{p}(P, Q)=(U, V)$ is used to force $(\mathrm{P}, \mathrm{Q})$ satisfy the conditions (5):

$$
U_{i, j, c}= \begin{cases}P_{i, j, c} / \max \left\{1, \sqrt{\sum_{c=1}^{C}\left(P_{i, j, c}^{2}+Q_{i, j, c}^{2}\right)}\right\} & \forall i, j, c \\ P_{i, n, c} / \max \left\{1, \sqrt{\sum_{c=1}^{C} P_{i, n, c}^{2}}\right\} & \forall i, c\end{cases}
$$

and

$$
V_{i, j, c}= \begin{cases}Q_{i, j, c} / \max \left\{1, \sqrt{\sum_{c=1}^{C}\left(P_{i, j, c}^{2}+Q_{i, j, c}^{2}\right)}\right\} & \forall i, j, c \\ Q_{m, j, c} / \max \left\{1, \sqrt{\sum_{c=1}^{C} Q_{m, j, c}^{2}}\right\} & \forall j, c\end{cases}
$$


Accelerated by FISTA [14, the convergence rate of Algorithm 2 is $\mathcal{O}\left(1 / k^{2}\right)$. The total time complexity of JTVMRI is $\mathcal{O}(C N \log N)$ by applying fast Fourier transform (FFT). There are double loops in the algorithm. Due to the trade off between efficiency and effect, the JTV denoising algorithm only runs 1 iteration in our implementation.

\section{Experiments}

\subsection{Materials and Methods}

The experiments are conducted on three brain MRI datasets shown in Figure 2. These images are widely used for validating pMRI methods [4] [7] [5]. Figure 2 (a) shows an image scanned from a GE $3 \mathrm{~T}$ commercial scanner with an eightchannel head coil using a two-dimensional T1-weighted spin echo protocol (TE $=11 \mathrm{~ms}, \mathrm{TR}=700 \mathrm{~ms}, \mathrm{FOV}=22 \mathrm{~cm}, 256 \times 256$ pixels $)$. Figure 2(b) shows a T1-weighted image from spoiled gradient echo (SPGR) sequence, scanned on a GE Signa-Excite 1.5-T scanner with an eight-channel receive coil ( $\mathrm{TE}=8 \mathrm{~ms}$, $\mathrm{TR}=17.6 \mathrm{~ms}, \mathrm{FOV}=20 \mathrm{~cm}, 200 \times 200$ pixels). The image shown in Figure 2(c) is a T1-weighted image extracted from SRI24 Multi-Channel Brain Atlas Data 16. It was acquired with a $3 \mathrm{D}$ axial IR-prep SPGR sequence, where $\mathrm{TR}=6.5 \mathrm{~ms}$ and $\mathrm{TE}=1.54 \mathrm{~ms}$. Imaging was performed on a $3.0 \mathrm{~T}$ GE scanner with an 8-channel head coil with $256 \times 256$ resolution covering a 24 -cm FOV.

Gaussian random sampling masks are used, with more samples are obtained in low frequencies and less samples are obtained in higher frequencies [7]. Our method is compared with the-state-of-the-art auto-calibration methods GRAPPA [3], SPIRiT [4] and the calibrationless CSMRI methods CaLMMRI [7] and CSSENSE [5]. All codes are downloaded from their websites and we carefully follow their experiment setup. For fair comparison, the sampling mask contains a $30 \times 30$ fully sampled region to ensure accurate calibration for GRAPPA and SPIRiT (shown in Figure 2(d)). All experiments are conducted in MATLAB with a $3.4 \mathrm{GHz}$ CPU. Gaussian white noise with 0.01 standard deviation is added in the original data and Signal-to-Noise Ratio (SNR) is used as the metric for evaluation. The parameters are set $\alpha=0.04, \beta=0.4$ for our algorithm and CSSENSE. All other algorithms are with their default parameters. SPIRiT runs 10 CG iterations as suggested because the result would be worse with more iterations [4]. All other algorithms run 50 iterations.

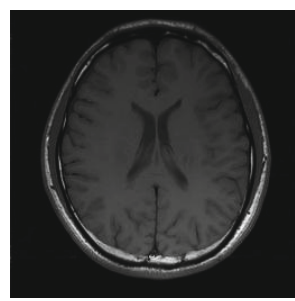

(a)

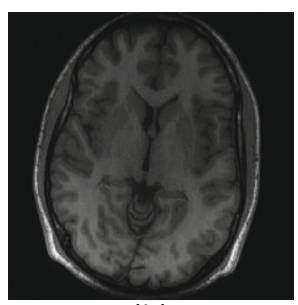

(b)

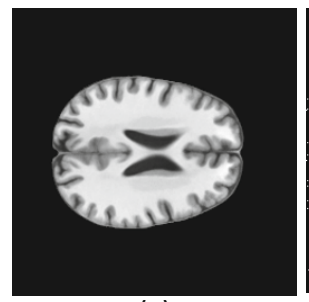

(c)

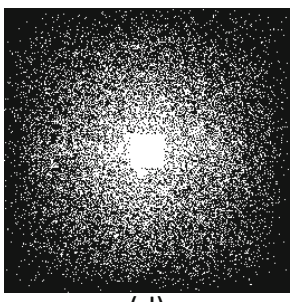

(d)

Fig. 2. Three MR brain images and the sampling mask 


\subsection{Results}

Figure 3 shows the reconstruction results on the first brain image at a reduction factor $R=4$. Compared to all others, the result of our method preserve most details of the original image (as shown in the zoomed region of interest). The results of other algorithms have visible artifacts while ours are the closest to the original one.

For better visualization of the difference errors, all the corresponding error images are shown in Figure 4. The error of our algorithm only appears slightly on the edges while those of all other algorithms significantly distribute over all the image. The SNRs of GRAPPA, SPIRiT, CSSENSE, CaLMMRI and the proposed method are $23.27,24.73,23.1 ., 22.21$ and 26.20 respectively. Their CPU time consumptions are $626.2 \mathrm{~s}, 7.8 \mathrm{~s}, 55.7 \mathrm{~s}, 12.5 \mathrm{~s}$ and $7.4 \mathrm{~s}$ respectively.

To reduce randomness, each algorithms run 100 times to obtain the average results. The average SNRs and CPU time on the three images are shown in Table 1 and Tabel 2 respectively. On each image, our method always achieve the best results with the least CPU time. GRAPPA is very slow on the random sampling mask, which need more than 10 minutes for a $256 \times 256$ image. Unlike other iterative methods, the SNR of SPIRiT does not increase monotonously 4]. How to choose the optimal iteration number to terminate is still unsolved. CaLMMRI is hard to be comparable to the state-of-the-art methods such as CSSENSE and SPIRiT. These results is consistent with the comparisons in previous work[7]. CSSENSE in our implementation is the same as coil-by-coil
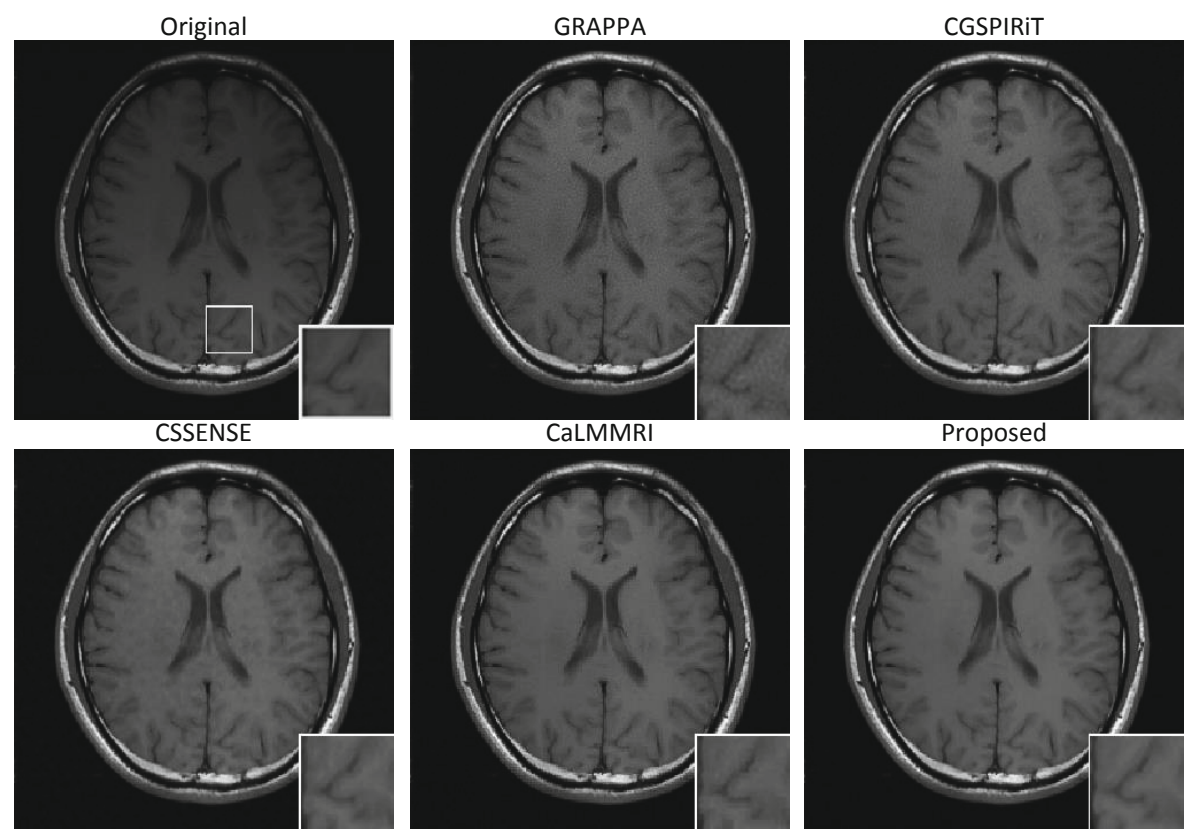

Fig. 3. Visual results of different methods with reduction factor $R=4$ 


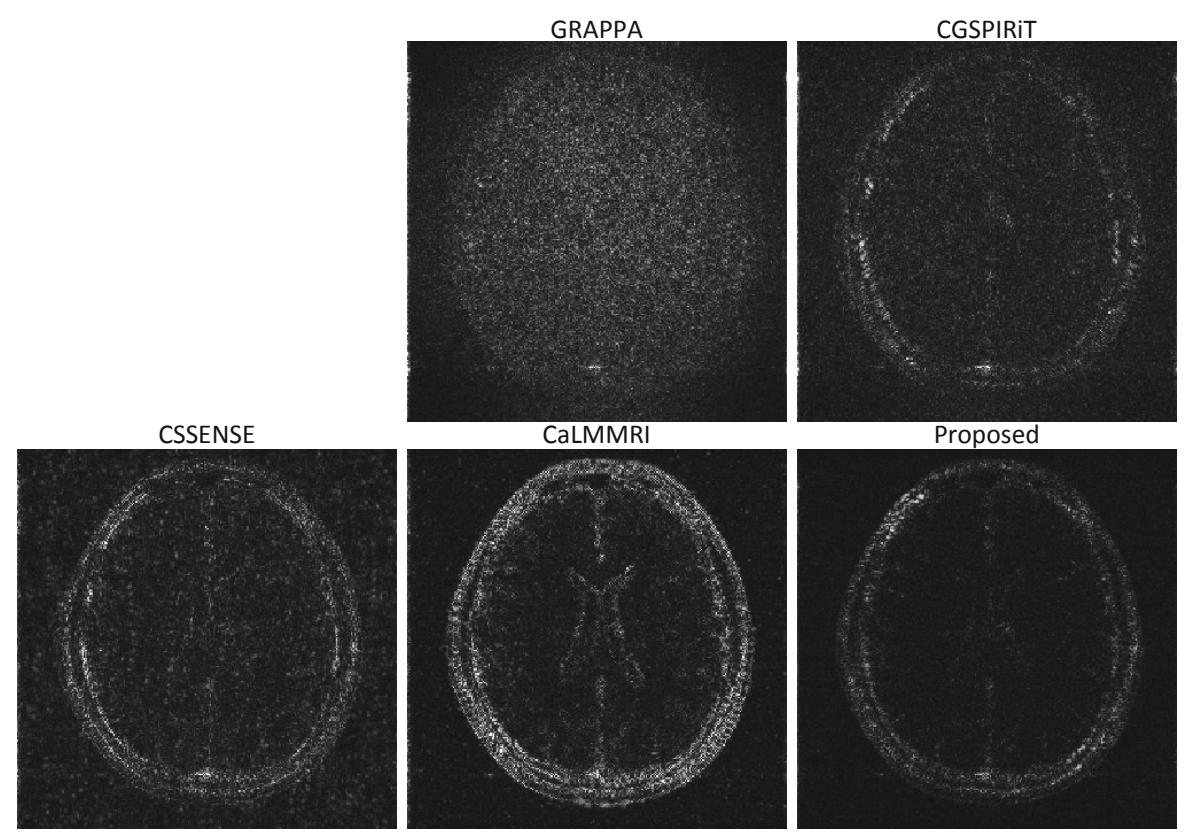

Fig. 4. The error images of different methods with reduction factor $R=4$

Table 1. The SNRs (dB) of different methods performed on $3 \mathrm{MR}$ images

\begin{tabular}{l|c|c|c|c|c}
\hline & GRAPPA [3] & CGSPIRiT [4] & CSSENSE[5] & CaLMMRI[7] & Proposed \\
\hline Brain 1 & 23.31 & 24.80 & 23.10 & 22.27 & $\mathbf{2 6 . 1 9}$ \\
Brain 2 & 18.39 & 20.85 & 21.17 & 20.28 & $\mathbf{2 2 . 9 9}$ \\
Brain 3 & 36.81 & 32.00 & 32.18 & 28.58 & $\mathbf{3 8 . 1 3}$ \\
\hline
\end{tabular}

Table 2. The CPU time (s) of different methods performed on 3 MR images

\begin{tabular}{l|c|c|c|c|c}
\hline & GRAPPA [3] & CGSPIRiT[4] & CSSENSE[5] & CaLMMRI[7] & Proposed \\
\hline Brain 1 & 588.5 & 7.2 & 58.4 & 10.1 & $\mathbf{6 . 5}$ \\
Brain 2 & 719.1 & 9.6 & 71.0 & 14.3 & $\mathbf{8 . 3}$ \\
Brain 3 & 723.8 & 9.4 & 71.5 & 14.9 & $\mathbf{9 . 1}$ \\
\hline
\end{tabular}

SparseMRI 8] reconstruction for each individual image. It has stable performance. However, the correlations among the aliased images are not exploited. That is why it always worse than the proposed method. The computational cost of CSSENSE is also expensive compared with other iterative methods, which makes it hard to be applied on large scale data.

\subsection{Discussion}

In contrast to GRAPPA and SPIRiT, CSSENSE and CaLMMRI are calibrationless methods that do not rely on sufficient ACS. In contrast to GRAPPA and 
CSSENSE, SPIRiT and CaLMMRI can perform joint reconstruction on the undersampled data. Compare with CaLMMRI, CSSENSE has utilized the sparsity in gradients to improve reconstruction. Only our method inherit all these benefits in pMRI, which can efficiently, jointly, reconstruct MR images by exploiting their sparsity in gradient domain without calibration.

\section{Conclusion}

In this paper, we have proposed a novel calibrationless method for calibrationless pMRI. It is motivated by the observation that the images of different coils are jointly sparse in the gradient domain. Compared to existing auto-calibration methods, our algorithms can be widely applied in the cases when calibration is time consuming or impossible. Compared to exiting calibrationless methods, our model is much more applicable on pMRI data and our algorithm is much more efficient. Extensive experiments have demonstrated that our algorithm outperforms the state-of-the-art auto-calibration methods when sufficient calibration can be achieved, and is far better than previous calibrationless methods. All these benefits make calibrationless pMRI much more feasible than before.

\section{References}

1. Pruessmann, K.P., Weiger, M., Scheidegger, M.B., Boesiger, P., et al.: SENSE: sensitivity encoding for fast MRI. Magn. Reson. Med. 42(5), 952-962 (1999)

2. Ying, L., Sheng, J.: Joint image reconstruction and sensitivity estimation in SENSE (JSENSE). Magn. Reson. Med. 57(6), 1196-1202 (2007)

3. Griswold, M.A., Blaimer, M., Breuer, F., Heidemann, R.M., Mueller, M., Jakob, P.M.: Parallel magnetic resonance imaging using the GRAPPA operator formalism. Magn. Reson. Med. 54(6), 1553-1556 (2005)

4. Lustig, M., Pauly, J.M.: SPIRiT: Iterative self-consistent parallel imaging reconstruction from arbitrary k-space. Magn. Reson. Med. 64(2), 457-471 (2010)

5. Liang, D., Liu, B., Wang, J., Ying, L.: Accelerating SENSE using compressed sensing. Magn. Reson. Med. 62(6), 1574-1584 (2009)

6. Zhao, T., Hu, X.: Iterative GRAPPA (iGRAPPA) for improved parallel imaging reconstruction. Magn. Reson. Med. 59(4), 903-907 (2008)

7. Majumdar, A., Ward, R.K.: Calibration-Less Multi-coil MR image reconstruction. Magn. Reson. Imaging (2012)

8. Lustig, M., Donoho, D., Pauly, J.: Sparse MRI: The application of compressed sensing for rapid MR imaging. Magn. Reson. Med. 58(6), 1182-1195 (2007)

9. Candès, E., Romberg, J., Tao, T.: Robust uncertainty principles: Exact signal reconstruction from highly incomplete frequency information. IEEE Trans. Inf. Theory 52(2), 489-509 (2006)

10. Donoho, D.: Compressed sensing. IEEE Trans. Inf. Theory 52(4), 1289-1306 (2006)

11. Acar, R., Vogel, C.R.: Analysis of bounded variation penalty methods for ill-posed problems. Inv. Probl. 10(6), 1217 (1999)

12. Van Den Berg, E., Friedlander, M.: Probing the pareto frontier for basis pursuit solutions. SIAM J. Sci. Comput. 31(2), 890-912 (2008) 
13. Bresson, X., Chan, T.F.: Fast dual minimization of the vectorial total variation norm and applications to color image processing. Inverse Problems and Imaging 2(4), 455-484 (2008)

14. Beck, A., Teboulle, M.: A fast iterative shrinkage-thresholding algorithm for linear inverse problems. SIAM J. Imaging Sci. 2(1), 183-202 (2009)

15. Beck, A., Teboulle, M.: Fast gradient-based algorithms for constrained total variation image denoising and deblurring problems. IEEE Trans. Image Process. 18(11), 2419-2434 (2009)

16. Rohlfing, T., Zahr, N.M., Sullivan, E., Pfefferbaum, A.: The SRI24 multichannel atlas of normal adult human brain structure. Human Brain Mapping 31, 798-819 (2010) 